\title{
Modelo de simulación de producción de materia seca y concentración de proteína bruta de gramíneas creciendo en bosques de Nothofagus antarctica (G. Forster) Oerst. bajo uso silvopastoril
}

\author{
Héctor A. Bahamonde ${ }^{1,2, \bowtie}$; Pablo L. Peri ${ }^{1,2,3} \&$ Juan P. Mayo ${ }^{2}$ \\ 1. Instituto Nacional de Tecnología Agropecuaria, Río Gallegos, Santa Cruz, Argentina. 2. Universidad Nacional de la \\ Patagonia Austral, Río Gallegos, Argentina. 3. Consejo Nacional de Investigaciones Científicas y Técnicas, Argentina.

\begin{abstract}
Resumen. Nothofagus antarctica (ñire) ocupa una importante fracción de los bosques de la Patagonia Austral y suele ser usado en sistemas silvopastoriles. Se carece, sin embargo de información que permita hacer un uso óptimo y sustentable de estos sistemas. En este trabajo se modeló la producción de materia seca (MS) y la variación en la concentración de proteína bruta $(\mathrm{PB})$ de gramíneas en sistemas silvopastoriles en bosques de ñire, considerando variables ambientales (temperaturas de aire y suelo, humedad de aire y suelo, calidad de sitio, cobertura de copas, entre otras) y aplicando información propia y existente. El modelo se focalizó a la escala de rodal; se consideró una población homogénea y coetánea de ñire bajo las condiciones del sur de la Patagonia (Santa Cruz y Tierra del Fuego). Un análisis de sensibilidad mostró que los parámetros más influyentes fueron la clase de sitio sobre MS y la temperatura del suelo sobre PB. Al comparar valores reales y simulados se encontró una correlación lineal significativa para MS $(\mathrm{R}=0.52 ; P<0.001$, subestimación media de $22 \%)$ y $\mathrm{PB}$ $(\mathrm{R}=0.47 ; \mathrm{P}<0.001$, sobreestimación media de $24 \%)$. Los resultados de las simulaciones son coherentes con los antecedentes empíricos respecto al efecto del ambiente y el manejo del rodal sobre la MS y PB del sotobosque y sugieren que el modelo es aceptable para ser usado como una herramienta orientadora del manejo de los pastizales en bosques de ñire.
\end{abstract}

[Palabras clave: sistemas silvopastoriles en Patagonia, bosque nativo, producción y calidad de forraje]

\begin{abstract}
AвSTRACT. Simulation model of dry matter production and crude protein concentration of grasses growing in Nothofagus antarctica (G. Forster) Oerst. forests under silvopastoral use: Nothofagus antarctica (ñire) occupies a significant fraction of the Southern Patagonian forests and are often used as silvopastoral systems. It lacks, however the information to make optimal and sustainable use of these systems. In this work we modeled the production of dry matter (DM) and the variation in the concentration of crude protein (CP) of grasses in silvopastoral systems in ñire forests, considering environmental variables (air and soil temperature, air humidity, soil moisture, site quality, crown cover, including) and applying existing and own information. The model was focused on the scale of stand considering a homogeneous and coeval population of ñire under conditions of southern Patagonia (Santa Cruz and Tierra del Fuego). Model sensitivity analysis showed that the most important parameter were the stand site quality on DM and the soil temperature on the $\mathrm{CP}$. We found a significant lineal correlation for $\mathrm{DM}(\mathrm{R}=0.52 ; P<0.001$, average underestimation $22 \%)$ and $\mathrm{CP}(\mathrm{R}=0.47 ; P<0.001$, average overestimation $24 \%$ ) when real and estimated values from the model were compared. The results of the simulations were coherent with empirical data in regard to the environmental variables and management of the stand affecting DM and CP of the understory, and suggest that the model is acceptable for use as a guiding tool in the management of understory grasses in ñire forests.
\end{abstract}

[Keywords: silvopastoral systems in Patagonia, native forest, forage production and quality]

\section{INTRODUCCIÓN}

En un sistema silvopastoril se pueden distinguir cuatro componentes principales: árboles, pasturas, animales y suelo (Mead 2009), sobre los cuales podemos intervenir para lograr una respuesta deseada. Al mismo tiempo, estos componentes interactúan entre sí y generan distintas respuestas según la intensidad de la intervención y las características ambientales de cada lugar (e.g., régimen hídrico, temperatura, topografía, etc.) (Fernández et al. 2002; Peri et al. 2005). Desde el punto de vista de la productividad y calidad

Editor asociado: Esteban Jobbágy

$\triangle$ bahamonde.hector@inta.gob.ar del pastizal, en un sistema silvopastoril las interacciones ecológicas con el componente arbóreo pueden ser positivas o negativas (Schroth 1999) y estarían dadas principalmente por la competencia por luz, agua y/o nutrientes. En este sentido, el uso silvopastoril de los bosques de Nothofagus antarctica (ñire) en Patagonia sur (Santa Cruz y Tierra del Fuego) ha sido muy estudiado desde distintas perspectivas ambientales y productivas (Peri et al. 2005; Bahamonde et al. 2009; Gargaglione 2011), lo cual ha generado una gran cantidad de información referida a relaciones parciales entre algunos componentes que inciden 
sobre la productividad y calidad forrajera del sistema silvopastoril. Sin embargo, no se ha desarrollado una herramienta que integre la información existente con un enfoque sistémico, es decir, que estudie las relaciones entre variables conectadas entre sí a través de relaciones causales, incluyendo las retroalimentaciones entre variables (Haraldsson 2000). Este enfoque sistémico permite también describir la dinámica (variación a través del tiempo) de las variables y/o sus relaciones (Sverdrup \& Haraldsson 2001), lo que posibilita una mejor comprensión de las interacciones existentes y una representación gráfica y funcional mediante software diseñado para la generación de modelos de simulación dinámicos. En el caso de los sistemas silvopastoriles en bosques de ñire, contar con una herramienta de este tipo permitiría describir asociaciones entre algunas de las principales condiciones ambientales que caracterizan el dosel de los bosques de ñire y el crecimiento y el valor nutritivo de su sotobosque graminoso. Al mismo tiempo, la información obtenida a través de esta herramienta puede ser de utilidad para reforzar las pautas de manejo existentes en el uso silvopastoril de bosques de ñire en Patagonia (Peri et al. 2009) a través de generar capacidad predictiva sobre la productividad y calidad del forraje en función de las condiciones ambientales y las prácticas de manejo bajo las que se desarrolle un bosque de ñire. El objetivo de este trabajo fue modelar la producción de materia seca y variación en la concentración de proteína bruta (PB) de gramíneas en sistemas silvopastoriles en bosques de ñire, usando las principales variables ambientales que tienen incidencia en estos sistemas.

\section{MATERIALES y MÉTODOS}

\section{Área de estudio}

El área de influencia de este trabajo incluye los bosques de ñire de Patagonia sur (Santa Cruz y Tierra del Fuego), que cubren una superficie aproximada de 400000 ha. Un $70 \%$ de esta superficie tiene uso silvopastoril (Peri \& Ormaechea 2013; Collado 2009). En Santa Cruz, más de $80 \%$ de estos bosques ocupa la porción sudoeste de la provincia en terrenos con pendientes menores a $5^{\circ}$, en el ecotono entre el bosque de lenga (Nothofagus pumilio) y la estepa (Peri \& Ormaechea 2013). Las precipitaciones anuales que no superan $500 \mathrm{~mm}$ y la temperatura media anual es de $5.2^{\circ} \mathrm{C}$ (Hijmans et al. 2005; www.worlclim.com). Similarmente en Tierra del Fuego, los bosques de ñire se ubican en su mayoría en la parte centro-Norte de la isla, en el límite con la estepa, en condiciones ambientales parecidas a las de Santa Cruz (Collado 2009).

\section{Desarrollo del modelo}

Se definió el propósito del modelo, sus límites, y se identificaron las variables claves del mismo. Además, se diagramaron las principales asociaciones del sistema sobre la base de las respuestas observadas a campo por diversos autores (Peri 2009; Bahamonde et al. 2012a). Para esto se usaron diagramas de causas circulares, según lo descrito por Haraldsson (2000). Se empleó el Software Vensim PLE Versión 5.9c.

Los diagramas de causas circulares se convirtieron en lenguaje gráfico de flujos y acumulaciones y se ajustaron los parámetros de las ecuaciones necesarias para la transformación del modelo cualitativo en cuantitativo a través de técnicas de ajuste lineal y no lineal (regresiones lineales simples y múltiples, regresiones no lineales, etc.). Se utilizó el soft STELLA, que simula la dinámica de los sistemas a través de flujos y acumulaciones. En Patagonia, el software se ha utilizado para modelar las interacciones en bosques nativos (Letourneau 2006) y en SSP en bosques implantados (Gyenge et al. 2006). Luego se establecieron los valores de los parámetros (o relaciones matemáticas) de cada relación generada en el modelo. Para ello se utilizaron datos obtenidos en el trabajo de Bahamonde et al. (2012a) sobre producción y calidad de gramíneas en bosques de ñire en la Patagonia argentina, y otra bibliografía relacionada. En este trabajo se presentarán las principales relaciones del modelo de producción de MS y PB desarrollado para gramíneas del sotobosque de sistemas silvopastoriles en bosques de ñire tomando como base el trabajo original realizado por Bahamonde (2011).

\section{Prueba del modelo}

En esta etapa, el modelo obtenido fue sometido a un análisis de sensibilidad para ver la magnitud y la dirección de la respuesta del modelo ante la modificación de algunos de los parámetros ajustados en la formulación del mismo (Breierova \& Choudhari 1996). Para ello se evaluaron las respuestas en producción de materia seca (MS) y porcentaje de PB del modelo ante modificaciones de $\pm 10 \%$ en los parámetros de las principales relaciones entre variables ambientales y productivas del modelo. Para cuantificar la sensibilidad del modelo se utilizó la siguiente fórmula:

$$
\% \text { de Sensibilización }=\frac{(\text { MSSensibilizado }-M S)}{M S} \times 100
$$

donde MS Sensibilizado es la producción de materia seca mensual de gramíneas simulada con algún parámetro sensibilizado (o modificado $\pm 10 \%$ ), y $M S$ es la producción de materia seca mensual de gramíneas simulada con los valores de los parámetros ajustados. De la misma manera se evaluó la sensibilidad del modelo para la concentración mensual de PB de las gramíneas; en esta etapa también se pusieron a prueba los resultados de la simulación. El modelo se validó con datos de estudios locales realizados por diferentes 
autores que se citarán oportunamente a lo largo del trabajo. Además, se desarrolló un estudio "ad hoc" entre octubre de 2010 y abril de 2011 para evaluar la producción de MS y la concentración de $\mathrm{PB}$ de diversas gramíneas del sotobosque en dos bosques de ñire, como se describe a continuación. Se utilizaron 2 rodales de ñire creciendo en calidades de sitio contrastantes de acuerdo a las diferentes clases de sitio (CS) establecidas por Ivancich et al. (2011). Estas CS distinguen las situaciones de producción de madera de los rodales de ñire a través de la altura de árboles maduros dominantes, categorizados en una escala de 1 a 5 ; estas diferentes calidades de sitio resultan de la combinación de clima, topografía y suelo (Skovsgaard \& Vanclay 2008). En Santa Cruz se utilizó un rodal ubicado en la Estancia Glen Cross $\left(51^{\circ} 50^{\prime} 15.4^{\prime \prime} \mathrm{S}-71^{\circ} 42^{\prime} 53.6^{\prime \prime} \mathrm{O}\right)$, correspondiente a una CS 5, con árboles dominantes de menos de 8 $m$ de altura que crecen en el límite con la estepa, con precipitaciones inferiores a $450 \mathrm{~mm} /$ año y temperatura media del aire en la temporada de crecimiento (septiembre-abril) de $7.6^{\circ} \mathrm{C}$. En Tierra del Fuego se evaluó un rodal en la Estancia San Pablo (54 $\left.15^{\prime} 40^{\prime \prime} \mathrm{S}-66^{\circ} 49^{\prime} 26^{\prime \prime} \mathrm{O}\right)$, perteneciente a una CS 2, con altura de árboles dominantes entre 12 y $14 \mathrm{~m}$, precipitaciones anuales de $550 \mathrm{~mm}$ y temperatura media del aire en la temporada de crecimiento de $7.4^{\circ} \mathrm{C}$.

En cada sitio se cuantificó la productividad (materia seca aérea) y el contenido de PB de gramíneas utilizando tres jaulas de clausura de $1.5 \times 1.2 \times 0.6 \mathrm{~m}$, tres jaulas por cada cobertura de copas en el bosque (bajo y entre copas), y tres jaulas en un sector aledaño sin cobertura arbórea. El material obtenido en cada corte fue secado en estufa a $60{ }^{\circ} \mathrm{C}$ hasta peso constante. Una submuestra de cada situación fue molida y enviada al laboratorio para la determinación de nitrógeno por el método semi-micro Kjeldhal, que al multiplicarlo por el coeficiente 6.25 permitió obtener su concentración de PB. Las gramíneas evaluadas fueron Agrostis perennans, Elymus magellanicus, E. angulatus, Bromus setifolius, B. catharticus, Dactylis glomerata, Deschampsia antarctica, D. flexuosa, Festuca gracillima, F. magellánica, F. pallescens, Holcus lanatus, Phleum alpinum, Poa pratensis, P. yagánica y Rytidosperma virescens. De esta manera, los valores de MS y $\mathrm{PB}$ obtenidos (en este trabajo y en bibliografía) fueron comparados con los que entrega el modelo simulando las condiciones ambientales de cada lugar.

Finalmente, a modo de ejemplo se realizaron simulaciones de producción de MS y concentración de PB de gramíneas en bosques de ñire en condiciones ambientales contrastantes, tratando de representar distintas ubicaciones de geográficas de Patagonia sur, y diferentes opciones de manejo del bosque de ñire. En Tierra del Fuego se simuló una situación en un bosque de ñire en una CS II y con una cobertura de copa de $90 \%$ que representaría un bosque sin manejo silvícola; de esta manera se puede comparar esta simulación con otras que incorporen remoción de árboles como práctica silvicultural tendiente al uso silvopastoril del bosque. En el caso de Santa Cruz, la simulación se basó en un bosque de ñire que crece en una CS IV con una cobertura de copas de $90 \%$ (apertura dosel 0.1). Luego, para los mismos sitios se simularon la producción de MS y el \%PB después de un raleo leve que deje un remanente de $70 \%$ de cobertura de copas (apertura dosel 0.3) y después de otro raleo más intenso que deje un remanente de sólo $30 \%$ de cobertura de copas (apertura dosel 0.7). Los valores mensuales de las variables ambientales (temperaturas de aire y suelo, y humedad volumétrica del suelo) fueron tomados de datos medidos en Bahamonde (2011) (Tabla S1, Información Suplementaria).

\section{Resultados}

\section{Conceptualización del modelo}

La representación de las relaciones entre los componentes ambientales del modelo y los componentes de crecimiento que representan acumulaciones como la biomasa de gramíneas y su concentración de $\mathrm{PB}$ reconocen su naturaleza circular (Figura S1, Información Suplementaria), en la que un elemento causal se vuelve afectado por el resultado de su efecto sobre otro componente del modelo. Por ejemplo, la biomasa acumulada será influenciada de manera positiva por su tasa de crecimiento. Sin embargo, dicho aumento de biomasa acumulada generará una disminución en la tasa de crecimiento. Por otro lado, el elemento que influye sobre la mayor cantidad de componentes del modelo es la apertura del dosel arbóreo. A diferencia de las otras relaciones del modelo, la relación entre la apertura del dosel arbóreo y la humedad del suelo no tiene signo porque el efecto puede ser positivo o negativo.

\section{Componentes del modelo y parametrización de sus relaciones}

Al observar los distintos componentes del modelo y las relaciones matemáticas que los vincularon entre sí (Apéndice, Información Suplementaria) se pudieron distinguir distintos aspectos que generaron la estructura del modelo. Los componentes estructurales del bosque de ñire (clase de sitio y apertura del dosel) se vincularon con la producción de MS del sotobosque de manera directa por un lado, e indirecta por otro a través del efecto que la estructura forestal genera sobre las variables ambientales (temperaturas de aire y suelo, humedad de suelo, radiación solar). A su vez, las variables ambientales inciden de forma directa sobre la productividad del pastizal. La concentración de PB del estrato graminoso fue afectada principalmente por 

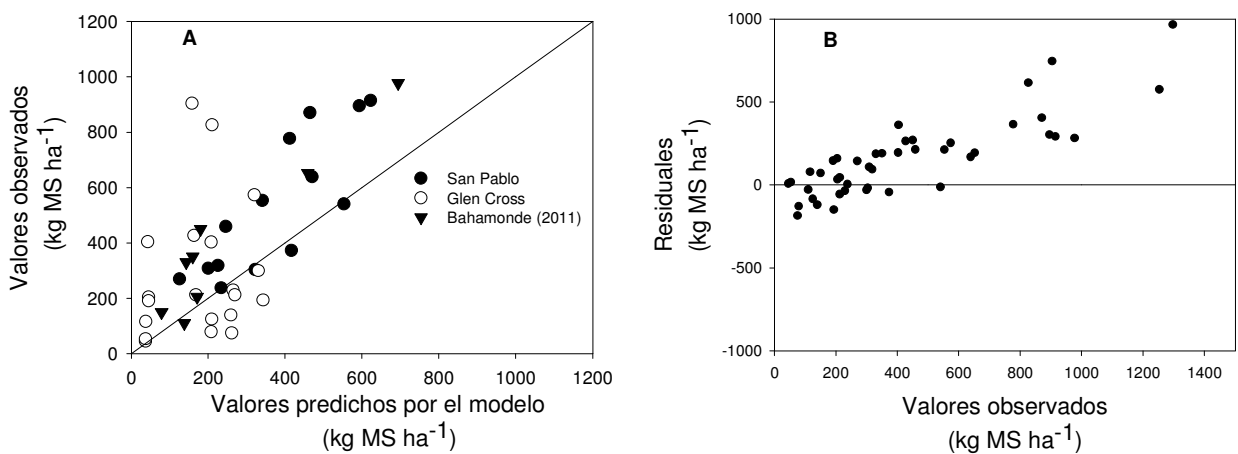

Figura 1. (A) Valores observados versus predichos por el modelo, de producción de materia seca de gramíneas creciendo en distintos bosques de N. antarctica de Patagonia. La línea representa la relación 1:1. (B) Residuales (observados menos predichos) versus valores observados.

Figure 1. (A) Observed versus predicted values of dry matter production of grasses growing at different $N$. antarctica forest in Patagonia. The line represents the 1:1 ratio. (B) Residuals (observed minus predicted values) versus observed values.

las variables ambientales de manera directa. Por último, la estructura del modelo incluyó el efecto del tiempo tanto para la producción de MS como para la concentración de PB.

\section{Prueba del modelo}

Análisis de sensibilidad. Para la producción de MS, este análisis mostró que los parámetros modificados $( \pm 10 \%)$ que mayor influencia tuvieron fueron aquellos relacionados a la CS (Apéndice, Ecuación 8), lo que generó una variación promedio de $27 \%$. Las restantes ecuaciones del modelo para producción de MS no superaron en promedio el $10 \%$ de sensibilidad ante modificaciones en igual magnitud de sus parámetros. En el caso del \%PB, la modificación de los parámetros generó menor variación en los resultados; la ecuación asociada al efecto de la temperatura del suelo (Apéndice 3, Ecuación 14) fue la más sensible, con una variación de $15.2 \%$.

Evaluación del modelo. Como se puede observar en la Figura 1A, el modelo logra

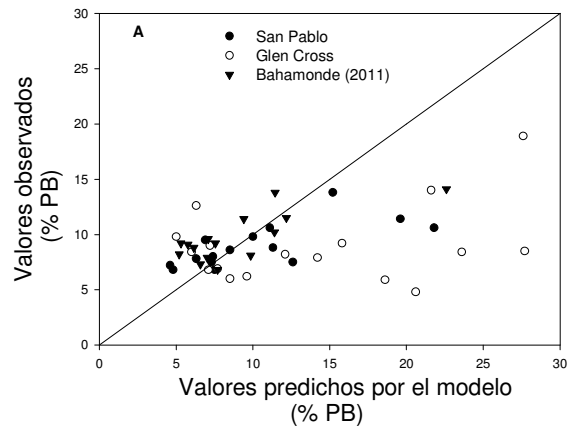

una correlación lineal significativa $(R=0.52$; $P<0.001)$ en la estimación de la MS. Por otro lado, el modelo presentó una tendencia a subestimar los valores de producción de materia seca respecto de los valores observados, con una subestimación media de $22 \%$. Esta tendencia aumenta cuando la producción de MS supera 600 kg MS/ha (Figura 1B), lo cual se condice con una correlación lineal positiva entre los valores observados de MS y los residuales (MS observada-MS estimada por el modelo) $(\mathrm{R}=0.90 ; \mathrm{P}<0.001)$.

De manera similar a lo ocurrido con la MS, los valores de PB estimados por el modelo se correlacionaron de forma positiva con los valores observados (Figura 2A) $(\mathrm{R}=0.47$; $P<0.001)$. En promedio, el modelo sobreestimó en $24 \%$ la concentración de PB respecto a los valores observados. Sin embargo, a diferencia de lo encontrado con la MS, no se encontró una tendencia que asocie dicha sobreestimación a los valores observados de PB, lo que fue coherente con una falta de correlación entre residuales y valores observados de PB (Figura 2B, P>0.05).

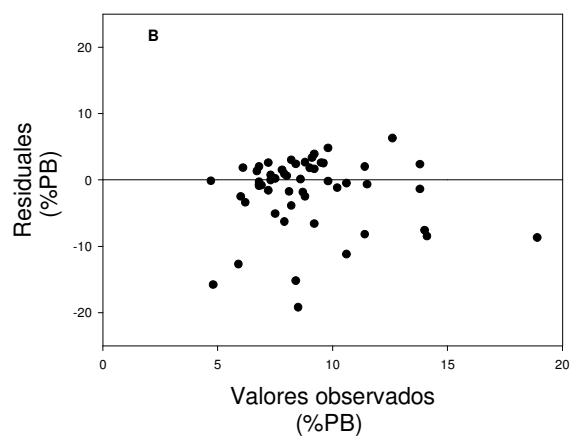

Figura 2. (A) Valores observados versus predichos por el modelo, de concentración de proteína bruta de gramíneas creciendo en distintos bosques de N. antarctica de Patagonia. La línea representa la relación 1:1. (B) Residuales (observados menos predichos) versus valores observados.

Figure 2. (A) Observed versus predicted values of crude protein content of grasses growing at different $N$. antarctica forest in Patagonia. The line represents the 1:1 ratio. (B) Residuals (observed minus predicted values) versus observed values. 

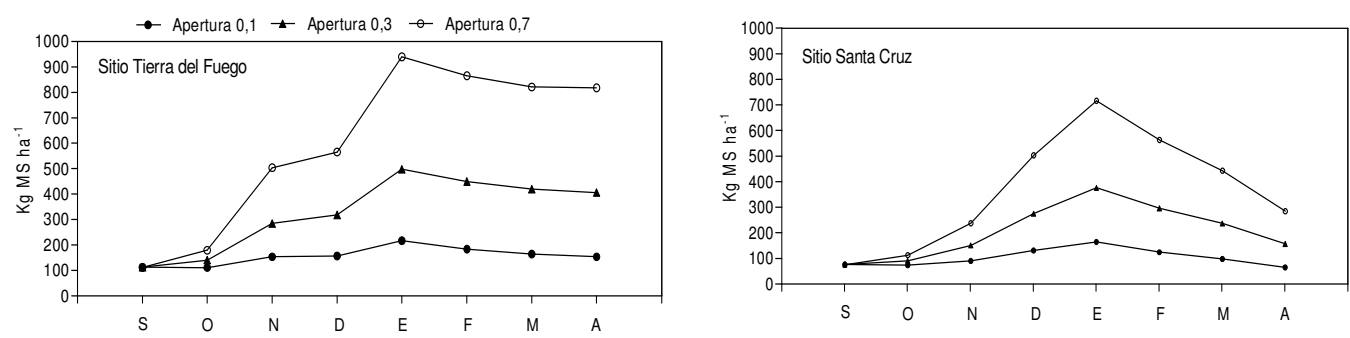

Figura 3. Variación en la producción de materia seca de gramíneas (kg MS/ha) durante una temporada de crecimiento, obtenidos a través del modelo de simulación, modificando la apertura del canopeo en bosques de ñire ubicados en Tierra del fuego y Santa Cruz.

Figure 3. Dry matter variation of grasses (kg MS/ha) during a growing season, obtained from a simulation model, modifying the canopy openness in ñire forests located in Tierra del Fuego and Santa Cruz.

La producción de materia seca obtenida a través de la simulación del modelo fue mínima en situaciones de alta cobertura arbórea (apertura 0.1), lo que representaría un bosque sin manejo, y alcanzó valores máximos de 217 y $164 \mathrm{~kg}$ MS/ha en Tierra del Fuego y Santa Cruz, respectivamente (ambos en enero; Figura 3). Asimismo, la simulación muestra que la producción de materia seca en ambos sitios aumenta a medida que se abre el dosel del bosque. El valor máximo de materia seca se estimó en el bosque que se desarrolla en la mejor CS (CS II, Tierra del Fuego) con 940 kg MS/ha vs. $717 \mathrm{~kg} / \mathrm{MS}$ ha en el sitio de Santa Cruz (CS IV). No obstante, las proporciones de las diferencias de producción de materia seca entre clases de sitio se ven amortizadas por las temperaturas más altas del sitio de Santa Cruz (Tabla S1). Por otro lado, la simulación muestra un comportamiento diferente entre sitios respecto de la variación temporal de la producción de MS, ya que si bien los valores más altos se alcanzan en enero en ambos sitios, en Tierra del Fuego la tasa de disminución es menor a la de Santa Cruz.

La simulación de la concentración de PB arrojó datos que no difirieron entre sitios (Figura 4), lo cual se debe a que el modelo no considera la CS en la que se desarrolla

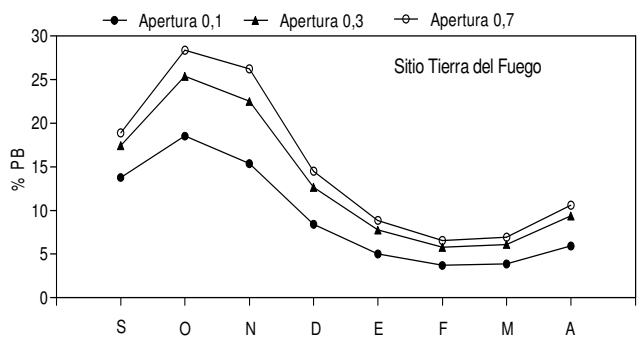

el bosque en la dinámica del \%PB. En los dos sitios simulados, la concentración de PB aumenta con la mayor apertura del canopeo, y alcanza valores máximos en la apertura de 0.7 , con valores de \%PB de 28.4 y 27.5 en CS II y IV, respectivamente. Por otro lado, la variación temporal de la concentración de PB muestra los valores más altos en los primeros meses de la temporada de crecimiento, para luego disminuir a valores mínimos con un leve aumento en la última etapa de crecimiento.

\section{DISCUSIÓN}

Un aporte importante del modelo obtenido es la posibilidad de visualizar de manera integrada distintos aspectos de los sistemas silvopastoriles de bosques de ñire. Por ejemplo, el efecto de la cobertura arbórea sobre variables ambientales (e.g., temperatura y humedad del suelo) según del nivel de cobertura de copas y de los valores de dichas variables en lugares adyacentes al bosque. Las relaciones descriptas conceptualmente y las ecuaciones obtenidas a través de datos empíricos y de bibliografía lograron representar los principales factores que determinan la variación temporal de la producción de MS y la concentración de PB de gramíneas. Por otro lado, si se tiene en cuenta que el uso actual de los bosques de ñire

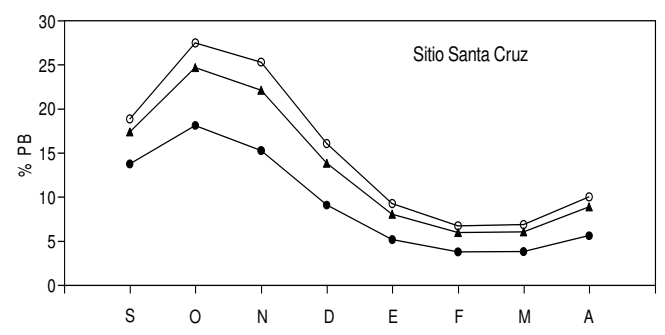

Figura 4. Variación en la concentración de proteína bruta de gramíneas (\%PB) durante una temporada de crecimiento, obtenidos a través del modelo de simulación, modificando la apertura del canopeo en bosques de ñire ubicados en Tierra del fuego y Santa Cruz.

Figure 4. Crude protein variation of grasses $(\% \mathrm{~PB})$ during a growing season, obtained from a simulation model, modifying the canopy openness in ñire forests located in Tierra del Fuego and Santa Cruz. 
como sistemas silvopastoriles en Santa Cruz y Tierra del Fuego no incorpora planificación ni herramientas de manejo (e.g., separación de ambientes, evaluación de pastizales o criterios de cálculo de carga animal) (Ormaechea et al., 2009), un modelo de este tipo podría contribuir a superar esas falencias mediante simulaciones bajo distintos escenarios de variación ambiental y manejo.

En general, los modelos de simulación para distintos aspectos de los sistemas silvopastoriles son escasos. Bergez et al. (1999) generaron un modelo de simulación para sistemas silvopastoriles en Europa; la principal virtud de ese modelo es que incorpora la mayoría de los componentes de estos sistemas (i.e., árboles, pastura, suelo, animales y clima) y sus relaciones biofísicas. Sin embargo, el modelo carece de especificidad ya que está basado en su mayoría en datos de literatura de distintas especies arbóreas y herbáceas. Asimismo, su validación es débil por no contar con muchas situaciones que permitan poner a prueba algunos aspectos a través del tiempo, como, por ejemplo, el crecimiento de los árboles. Gillet et al. (2002) modelaron la dinámica del estrato herbáceo de sistemas silvopastoriles a nivel de comunidad en sistemas extensivos que podrían parecerse más a la escala en la que se dan los sistemas silvopastoriles con bosque de ñire en Patagonia. Sin embargo, las relaciones entre los componentes bióticos son más complejas debido a que incluyen árboles, arbustos y pastizales de distintos niveles nutricionales. En Nueva Zelanda, Knowles et al. (1999) generaron un modelo de predicción de producción de materia seca del sotobosque en función de la cobertura de copas en una plantación de Pinus ponderosa, mostrando una alta correlación entre ambas variables. No obstante, ese modelo tendría una aplicación acotada al lugar donde fue generado. En el nivel regional, sólo se conoce un modelo desarrollado en Patagonia Norte para crecimiento de Festuca pallescens en sistemas silvopastoriles en bosques implantados de Pinus ponderosa (Gyenge et al. 2006), en el cual se simuló la producción de MS anual. Sin embargo, dicho modelo no considera la variación mensual ni aspectos de calidad del pastizal. Teniendo en cuenta estos antecedentes se destaca la especificidad de nuestro modelo, ya que está basado en relaciones empíricas obtenidas de estudios en bosques de ñire y en Patagonia sur. Así mismo, la simplicidad relativa de su estructura y su acotada escala temporal y espacial permiten ponerlo a prueba repetidas veces, y de esta manera mejorar los parámetros que relacionan matemáticamente las distintas variables. Otro aporte de este modelo es la incorporación de aspectos de calidad del estrato forrajero que pocas veces son considerados en este tipo de trabajos. No obstante, este modelo también presenta ciertas limitaciones que deben ser tenidas en cuenta. La principal corresponde a la humedad del suelo, variable planteada como un compartimento cerrado influenciado únicamente por el grado de apertura del dosel [lo cual, se sabe, es una simplificación que no contempla el dinamismo del agua en el suelo y su interacción con la vegetación en sistemas boscosos (Echeverría et al. 2007)]. Otro aspecto ausente en este modelo, y que ha sido incorporado en otros modelos de calidad de forraje, es la disponibilidad de nitrógeno en el suelo (Gustavsson et al. 1995). Para salvar estas limitaciones y mejorar el modelo se están iniciando estudios sobre la dinámica del agua en sistemas silvopastoriles con bosques de ñire y se pretende incorporar datos obtenidos en estos ambientes con respecto a los procesos relacionados con el $\mathrm{N}$ en el suelo (Bahamonde et al. 2012b; Bahamonde et al. 2013). Teniendo en cuenta los puntos anteriores se considera que el modelo de simulación obtenido en este trabajo constituye una herramienta importante para el manejo y el estudio de los sistemas silvopastoriles en bosques de ñire de Patagonia sur.

Agradecimientos: Los autores agradecen sinceramente la valiosa colaboración de Federico Letorneau en el uso del software para la generación del modelo, así como también a Roberto Álvarez y Atilio Barneix por sus aportes en la elaboración del trabajo.

\section{BiBLIOGRAFÍA}

Bahamonde, HA; PL Peri; G Martínez Pastur \& V Lencinas. 2009. Variaciones microclimáticas en bosques primarios y bajo uso silvopastoril de Nothofagus antarctica en dos Clases de Sitio en Patagonia sur. Primer congreso silvopastoril. Posadas, Misiones, Argentina. Pp. 289-296.

BAHAMONDE, HA. 2011. Efecto de variables ambientales sobre la Productividad Primaria Neta Aérea y la concentración de proteína bruta de gramíneas en Sistemas Silvopastoriles de ñire (Nothofagus antarctica): creación de un modelo de simulación. Tesis Maestría en Recursos Naturales Universidad de Buenos Aires (UBA). Pp. 148.

Bahamonde, HA; PL Peri; R Álvarez \& A Barneix. 2012a. Producción y calidad de gramíneas en un gradiente de calidades de sitio y coberturas en bosques de Nothofagus antárctica (G. Forster) Oerst. en Patagonia. Ecología Austral, 22:62-73.

Bahamonde, HA; PL Peri; R Álvarez; A barneix; A MORETTO; ET AL. 2012b. Litter decomposition and nutrients dynamics in Nothofagus antarctica forests under silvopastoral use in Southern Patagonia. Agroforestry Systems, 84:345-360. 
Bahamonde, HA; PL Peri; R Álvarez; A Barneix; A Moretto ; ET AL. 2013. Silvopastoral use of Nothofagus antarctica in Southern Patagonian forests, influence over net nitrogen soil mineralization. Agroforestry Systems, 87:259-271.

Bergez, JE; M EtIENNE \& P BALANDIER. 1999. ALWAYs: a plotbased silvopastoral system model. Ecological modeling, 115:1-17.

Breierova, L \& M Choudhari. 1996. An introduction to sensitivity analysis, MIT System Dynamics in Education Project. Pp. 67. http://ocw.mit.edu/courses/sloanschool-of-management/15-988-system-dynamicsself-study-fall-1998-spring-1999/readings / sensitivityanalysis.pdf

Collado, L. 2009. Informe final ordenamiento de bosques Ley 26331. Secretaría de Desarrollo Sustentable y Ambiente, Dirección general de Bosques, Tierra del Fuego. Pp. 13.

Echeverría, C; A Hubert \& F Taberlet. 2007. Estudio comparativo de los componentes del balance hídrico en un bosque nativo y una pradera en el sur de Chile. Bosque, 28:271-280.

FernándeZ, M; J Gyenge; G Dalla Salda \& T Schlichter. 2002. Silvopastoral systems in northwestern Patagonia. I: growth and photosynthesis of Stipa speciosa under different levels of Pinus ponderosa cover. Agroforestry Systems, 55:27-35.

Gargaglione, V. 2011. Dinámica de macronutrientes en bosques de Nothofagus antarctica de Patagonia sur. Tesis Doctorado en Ciencias Agrarias Universidad de Buenos Aires (UBA). Pp. 132.

Gillet, F; O Besson \& JM Gobat. 2002. patumod: a compartment model of vegetation dynamics in wooded pasture. Ecological Modelling, 187:267-290.

Gustavsson, AM; JF Angus \& BRW Torsell. 1995. An integrated model for growth and nutritional value of timothy. Agricultural Systems, 47:73-92.

Gyenge, J; C Carranza; ME Fernández; M Ledesma \& T SCHLICHTER. 2006. Desarrollo de un modelo de simulación de sistemas silvopastoriles (MSSP INTA). La Serena: Chile. 2006. Artículo Completo. II Congreso Latinoamericano IUFRO, IUFROLAT 2006.

Haraldsson, HV. 2000. Introduction to systems and causal loop diagrams. System Dynamic course. Lumes. Lund University. http://goo.gl/g3kX4b.

Hijmans, RJ; SE CAMERON; JL PARRa; PG Jones \& A Jarvis, 2005. Very high resolution interpolated climate surfaces for global land areas. International Journal of Climatology, 25:1965-1978.

Ivancich, H; G Martínez Pastur \& PL Peri. 2011. Modelos forzados y no forzados para el cálculo de índice de sitio en bosques de Nothofagus antarctica en Patagonia sur. Bosque, 32:135-145.

Knowles, RL; GC Horvath; MA Carter \& MF Hawke. 1999. Developing a canopy closure model to predict overstorey/understorey relationships in Pinus radiate silvopastoral systems. Agroforestry Systems, 43:109-119.

LETORNEAU, FJ. 2006. Estudio de las interacciones positivas y negativas sobre el crecimiento de Austrocedrus chilensis durante una etapa inicial de desarrollo, en un matorral sucecional mésico. Tesis Doctoral Universidad Nacional del Comahue, Argentina. Pp. 182.

Martínez Pastur, G; MV Lencinas; P Peri; A Moretto; JM Cellini; ET AL. 2007. Harvesting adaptation to Biodiversity Conservation in Sawmill Industry: Technology Innovation and Monitoring Program. Journal of Technology Management E Innovation, 2:58-70.

MCCALL, DG \& GJ Bishop-HuRLey. 2003. A pasture growth model for use in a whole-farm dairy production model. Agricultural Systems, 76:1183-1205.

MEAD, DJ. 2009. Biophysical interactions in silvopastoral systems: a New Zealand perspective. Pp. 3-9 en: Actas del $1^{\circ}$ Congreso Nacional de Sistemas Silvopastoriles. 14-16 Mayo, Misiones, Argentina.

Ormaechea, SG; PL Peri; R Molina \& JP Mayo. 2009. Situación y manejo actual del sector ganadero en establecimientos con bosque de ñire (Nothofagus antarctica) de Patagonia sur. Pp 385-393 en: Actas del $1^{\circ}$ Congreso Nacional de Sistemas Silvopastoriles. 14-16 Mayo, Misiones, Argentina.

Peri, PL; MV Sturzenbaum; L Monelos; E Livraghi; R Christiansen; et AL. 2005. Productividad de sistemas silvopastoriles en bosques nativos de ñire (Nothofagus antarctica) de Patagonia Austral. Actas III Congreso Forestal Argentino y Latinoamericano. Corrientes, Argentina.Pp. 10

Peri, P. 2009. Evaluación de Pastizales en bosques de Nothofagus antarctica - Método Nirantal sur. Pp. 335-342 en: Actas Primer Congreso Nacional Sistemas Silvopastoriles. Posadas, Misiones, Argentina.

Peri, PL; N Hansen; V Rusch; L Tejera; L Monelos; et AL. 2009. Pautas de manejo de sistemas silvopastoriles en bosques nativos de Nothofagus antarctica (ñire) en Patagonia. Pp. 151-155 en: Actas Primer Congreso Nacional Sistemas Silvopastoriles. Posadas, Misiones, Argentina.

Peri, P \& RG LASAgno. 2010. Biomass, carbon and nutrient storage for dominant grasses of cold temperate steppe grasslands in southern Patagonia, Argentina. Journal of Arid Environments, 74:23-34.

Peri, PL \& SG Ormaechea. 2013. Relevamiento de los bosques nativos de ñire (Nothofagus antarctica) en Santa Cruz: base para su conservación y manejo. Ediciones INTA. Pp. 88. ISBN 978-987-679-219-6.

Romera, AJ; DG McCall; JM Lee \& MG Agnusdei. 2009. Improving the McCall herbage growth model. New Zealand Journal of Agricultural Research, 52:s477-494.

Sснготн, G. 1999. A review of belowground interactions in agroforestry, focusing on mechanisms and management options. Agroforestry Systems, 43:5-34.

SkovsgaArd, JP \& JK VAnKLay. 2008. Forest site productivity: a review of the evolution of dendrometric concepts for even-aged stands. Forestry, 81:13-31.

SVERDRUP, HU \& HV HARALDSSON.2001. Finding simplicity in complexity in biogeochemical modelling. Pp. 430 en: Wainwright \& Mulligan (eds.). Environmental Modelling. John Wiley \& sons.

TAiz, L \& E Zeiger. 2006. Mineral nutrition. Pp 74-84 en: Plant physiology, 4th ed. Sinauer Associates Inc, Sunderland. 


\section{INFORMACIÓN SUPLEMENTARIA}

Tabla S1. Condiciones ambientales mensuales de dos rodales de bosque de ñire ubicados en Tierra del Fuego y Santa Cruz usados como ejemplopor un modelo de simulación de producción de materia seca y concentración de PB de gramíneas en bosques de ñire bajo uso silvopastoril.

Table S1. Monthly environmental conditions in two stands of ñire forest located in Tierra del Fuego and Santa Cruz, which are used as example by a simulation model of dry matter production and crude protein concentration of grasses in ñire forest under silvopastoral use.

\begin{tabular}{lcccccc}
\hline & \multicolumn{3}{c}{ Tierra del Fuego } & \multicolumn{3}{c}{ Santa Cruz } \\
\hline Mes & $\begin{array}{c}\text { Temp. } \\
\text { media aire }\left({ }^{\circ} \mathrm{C}\right)\end{array}$ & $\begin{array}{c}\text { Temp. } \\
\text { media suelo } \\
\left({ }^{\circ} \mathrm{C}\right)\end{array}$ & $\begin{array}{c}\text { Humedad } \\
\text { volumétrica } \\
\text { suelo }(\%)\end{array}$ & $\begin{array}{c}\text { Temp. } \\
\text { Media del aire } \\
\left({ }^{\circ} \mathrm{C}\right)\end{array}$ & $\begin{array}{c}\text { Temp. } \\
\text { media suelo } \\
\left({ }^{\circ} \mathrm{C}\right)\end{array}$ & $\begin{array}{c}\text { Humedad } \\
\text { volumétrica } \\
\text { suelo }(\%)\end{array}$ \\
Septiembre & 2,6 & 3,4 & 35,0 & 3,6 & 3,0 & 30,0 \\
Octubre & 4,4 & 5,3 & 58,4 & 4,6 & 4,1 & 42,8 \\
Noviembre & 6,6 & 7,7 & 11,6 & 7,3 & 7,1 & 45,7 \\
Diciembre & 8,2 & 10,8 & 24,7 & 10,8 & 9,1 & 26,0 \\
Febrero & 11,2 & 12,8 & 12,1 & 12,5 & 11 & 12,2 \\
Marzo & 12,1 & 13,4 & 13,3 & 14,2 & 14,6 & 11,6 \\
Abril & 8,4 & 10,3 & 21,2 & 9,5 & 9,4 & 10 \\
\hline & 4,9 & 6 & 30,2 & 5,9 & 4,9 & 16,2 \\
\hline
\end{tabular}

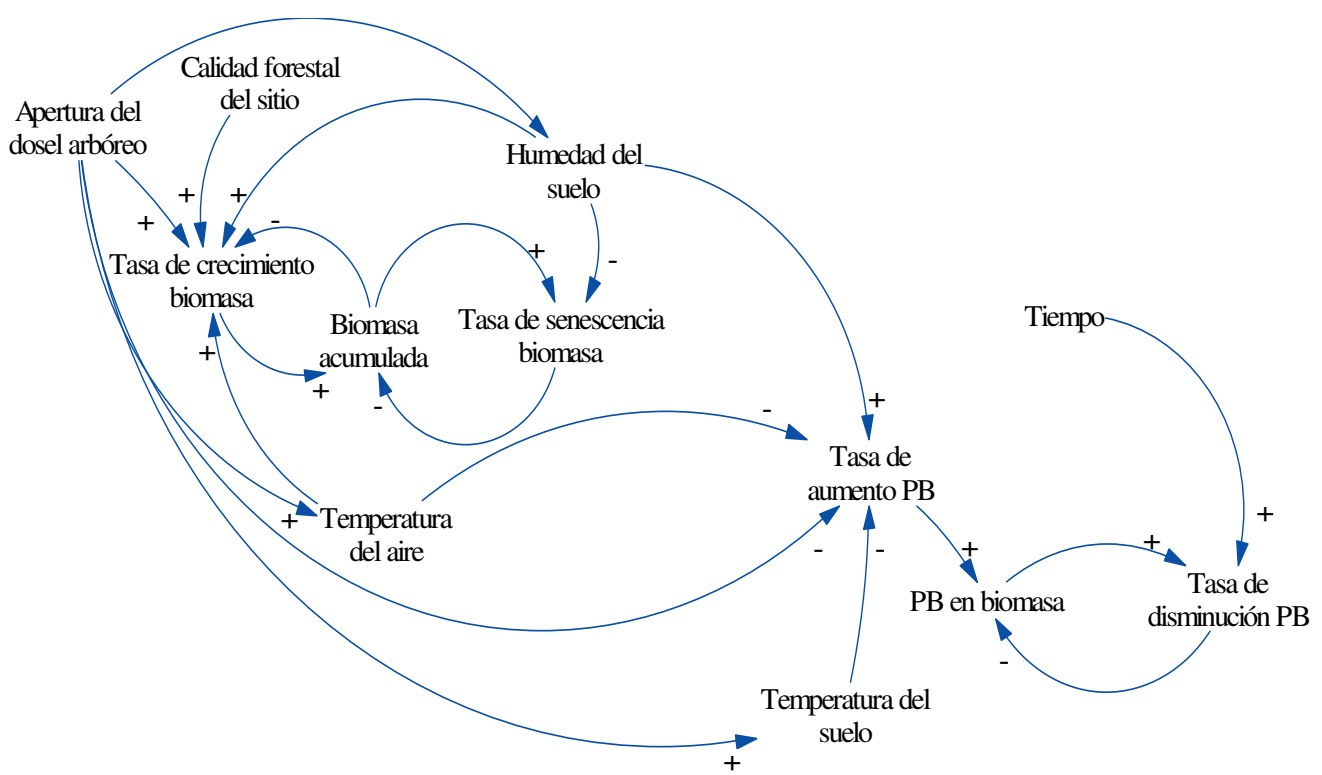

Figura S1. Diagrama de causas circulares que describe las relaciones entre los elementos del modelo de simulación de crecimiento de materia seca y concentración de proteína bruta de gramíneas en sistemas silvopastoriles de Nothofagus antarctica.

Figure S1. Loops diagram describing the relationship between different components in a simulation model of dry matter growing and crude protein concentration of grasses in a silvopastoral system with Nothofagus antarctica. 
Abril de 2014 MODELIZACIÓN DE MATERIA SECA Y CALIDAD DE GRAMÍNEAS EN BOSQUES DE ÑIRE

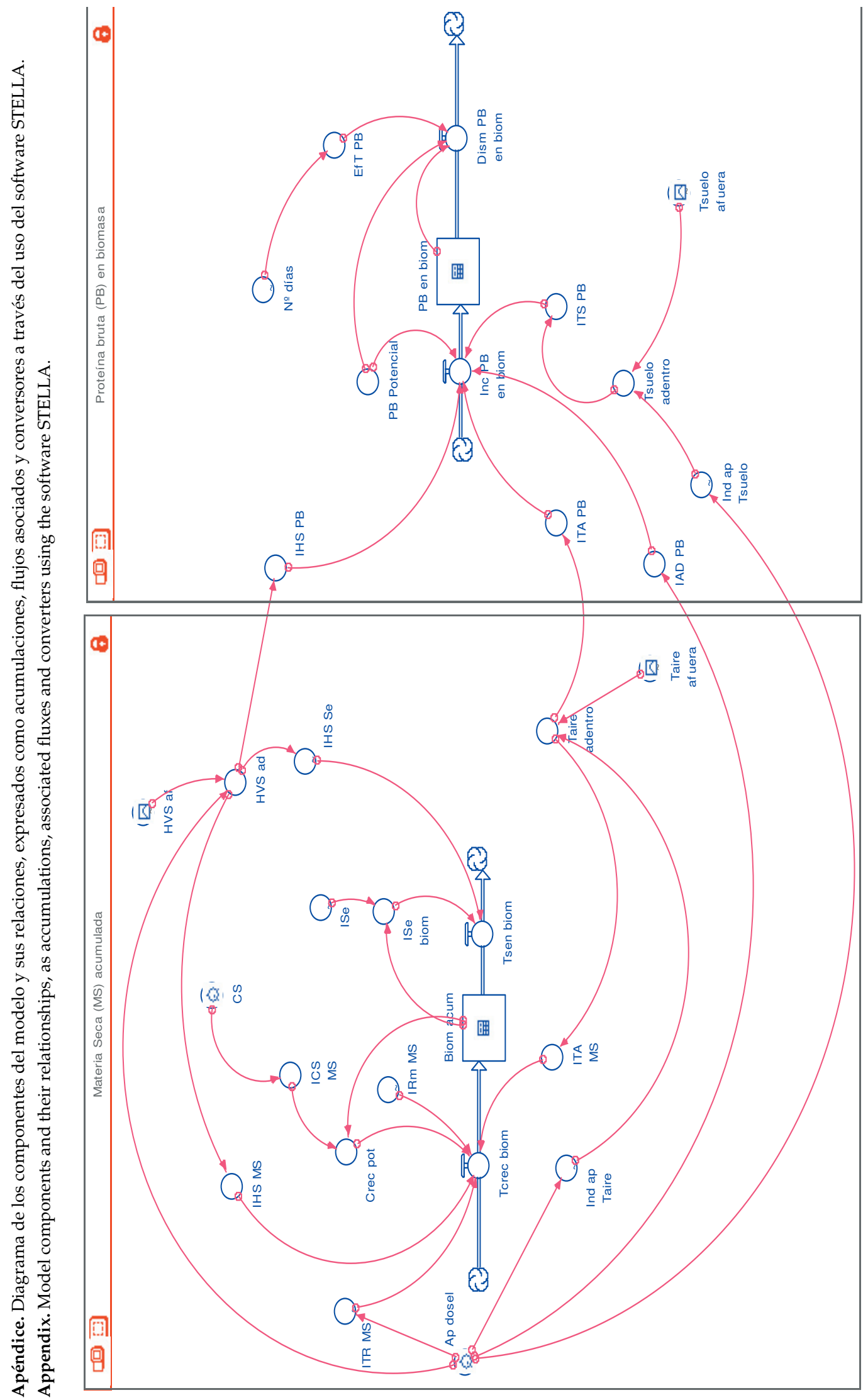


Componentes del modelo y parametrización de sus relaciones

Clase de Sitio (CS)

Está dado por la calidad de sitio del rodal en el que se desarrolla el sistema silvopastoril. Se usa la clasificación propuesta por Ivancich et al. (2011) para bosques de ñire que establece 5 clases de sitio (valores entre 1 y 5 ).

Apertura del dosel (Ap dosel)

Depende de la transmisividad lumínica dentro del bosque con respecto a un lugar sin árboles y varía desde 0,1 (cobertura arbórea del $90 \%$ ) a 1 (lugar sin árboles).

Número de días ( $\mathrm{N}^{\mathrm{o}}$ días)

Da información del número de días transcurridos desde el inicio hasta el fin de la temporada de crecimiento de gramíneas en los SSP.

\section{Temperatura del aire afuera (Taire afuera)}

Es la temperatura media mensual del aire en la temporada de crecimiento del pastizal. Para el modelo se deben ingresar los valores de temperatura en lugares sin árboles adyacentes al SSP. El modelo no toma valores negativos, ya que en su área de aplicación en la temporada de crecimiento no presenta valores negativos (Martínez Pastur et al. 2007; Bahamonde et al. 2012a).

Influencia de la apertura en la temperatura del aire (Indap Taire)

Índice que relaciona las temperaturas medias mensuales del aire fuera y dentro del bosque bajo distintas coberturas de copa. Toma valores entre 0,93 y 1 según la apertura del dosel del bosque (Bahamonde 2011).

Ind ap Taire $=0,97$ sí apertura dosel $\leq 0,3$

Ind ap Taire $=0,95$ sí apertura dosel $\geq 0,4 \leq 0,6$

Ind ap Taire $=0,93$ sí apertura dosel $\geq 0,7 \leq 0,8$

Ind ap Taire $=1$ sí apertura dosel $\geq 0,9$

Estos índices fueron generados de regresiones lineales simples obtenidas a partir de mediciones de temperatura de aire realizadas en distintos sitios de Santa Cruz y Tierra del Fuego durante dos años (Bahamonde et al. 2012a), de los cuales se tomaron los datos medidos durante el periodo de crecimiento (septiembre - abril).
A continuación se presentan las regresiones lineales simples obtenidas.

$$
\begin{aligned}
& Y_{1}=1,007629 X-0,199734 \mathrm{p}<0,001 \quad \mathrm{R}^{2}=0,99 \\
& Y_{2}=0,958383 X-0,078526 \mathrm{p}<0,001 \quad \mathrm{R}^{2}=0,97 \\
& Y_{3}=0,97936 X-0,266317 \mathrm{p}<0,001 \quad \mathrm{R}^{2}=0,99
\end{aligned}
$$

Donde $X$ : temperatura media mensual del aire a una apertura de canopeo de 1 (sin árboles); $Y_{1^{\prime}}, Y_{2^{\prime}}, Y_{3}$ : son temperaturas medias mensuales del aire a aperturas de canopeo de 0,$3 ; 0,5$ y 0,7 , respectivamente.

Temperatura media del aire adentro (Taire adentro)

Estima la temperatura media mensual del aire dentro del bosque a partir de la temperatura del aire en lugares adyacentes sin cobertura arbórea (ec. 1).

Taire adentro = Taire afuera ${ }^{*}$ Ind ap Taire [1]

Temperatura media del suelo afuera (Tsuelo afuera)

Temperatura media del suelo a $3 \mathrm{~cm}$ de profundidad durante la temporada de crecimiento en los lugares sin cobertura arbórea. El modelo trabaja con valores mayores o iguales a $0^{\circ} \mathrm{C}$ de acuerdo a los datos obtenidos en las zonas de estudio (Bahamonde et al. 2012a).

Influencia de la apertura en la temperatura del suelo (Ind apTsuelo)

Este índice relaciona las temperaturas del suelo en lugares sin cobertura arbórea con aquellas dentro del bosque. Toma valores de entre 0,8 y 1,2 según la apertura del dosel (Bahamonde 2011), como se muestra a continuación.

Ind ap Tsuelo $=0,8$ sí apertura dosel $\leq 0,3$

Ind ap Tsuelo $=1,2$ sí apertura dosel $\geq 0.4 \leq 0,6$

Ind ap Tsuelo $=0,9$ sí apertura dosel $\geq 0.7 \leq 0,8$

Ind ap Tsuelo $=1$ sí apertura dosel $\geq 0,9$

Los índices generados se obtuvieron de la misma manera que para la temperatura del aire. A continuación se muestran las regresiones lineales simples resultantes de las mediciones.

$$
\begin{aligned}
& Y_{1}=0,27274 X+2,70953 \mathrm{p}<0,001 \quad \mathrm{R}^{2}=0,94 \\
& Y_{2}=0,895142 X+1,28284 p<0,001 \quad R^{2}=0,94
\end{aligned}
$$


$Y_{3}=0,741263 X+0,83001 p<0,001 \quad R^{2}=0,81$

Donde $X$ : temperatura media mensual del suelo a una apertura de canopeo de $1 ; Y_{1}, Y_{2}$ $Y_{3}$ : son temperaturas medias mensuales del suelo a aperturas de canopeo de 0,$3 ; 0,5$ y 0,7, respectivamente.

Temperatura media del suelo adentro (Tsuelo adentro)

Temperatura media mensual del suelo dentro del bosque, obtenidos de la temperatura del suelo en lugares fuera del bosque por un factor que representa el efecto de la cobertura arbórea (ec. 2).

Tsuelo adentro $=$ Tsuelo afuera $*$ Ind ap Tsuelo [2]

Humedad volumétrica del suelo adentro (HVS ad)

Estima la humedad volumétrica del suelo (hasta $30 \mathrm{~cm}$ de profundidad) dentro del bosque (en distintas coberturas arbóreas) al inicio de cada mes de la temporada de crecimiento. Los valores se obtienen según la ecuación 3:

HVSad $=[((-0,007731 *(1,18741-$ Apdosel $) \quad *$ $\left.(\text { HVSaf })^{2}\right)+(1,04741 *$ HVSaf $)+((2,8985 *(1$ - Apdosel))]

Donde Apdosel es la apertura del dosel y HVSaf es la humedad volumétrica del suelo fuera del rodal. Esta ecuación se obtuvo a través de mediciones humedad volumétrica

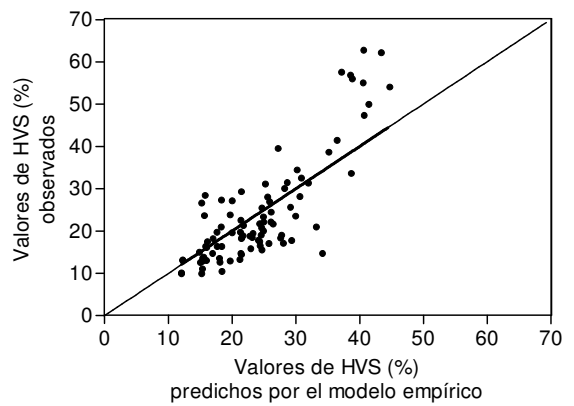

Figura S2. Relación entre valores de humedad volumétrica de suelo (HVS) a $30 \mathrm{~cm}$ de profundidad observados en bosques de ñire de Santa Cruz y Tierra del Fuego y los valoresestimadosporelmodeloexplicitadoenlaEcuación4. La línea representa la relación 1:1. La relación lineal entre valores observados y predichos es significativa $(P<0.001$; $\mathrm{R}^{2}=0.67$ ).

Figure S2. Relationship between observed and estimated (Equation 4) values of volumetric soil moisture in ñire forests located in Santa Cruz and Tierra del Fuego. The line represents the 1:1 relationship. The linear relationship between observed and predicted values is significant $\left(P<0.001 ; \mathrm{R}^{2}=0.67\right)$. del suelo en distintas coberturas arbóreas en dos bosques de ñire de la provincia de Santa Cruz (Bahamonde 2011). Para validar el modelo empírico obtenido se utilizaron datos de humedad volumétrica del suelo de Tierra del Fuego y Santa Cruz (Bahamonde et al. 2012a) y fueron comparados con los valores estimados por la ecuación (3) (Figura S2).

Biomasa acumulada (Biomacum)

Se refiere a la MS aérea de gramíneas acumulada mensualmente ( $\mathrm{kg}$ MS ha $\left.{ }^{-1}\right)$ durante la temporada de crecimiento. La variación mensual ( $\mathrm{t}$ ) de biomasa acumulada estará dada por la diferencia entre la tasa de crecimiento y tasa de senescencia durante el periodo (dt) (ec. 4).

Biomacum $(t)=$ Biomacum $(t-d t)+($ Tcrecbiom Tsenbiom $) * d t$

Para iniciar las corridas mensuales el modelo necesita un valor a partir del cual crecer. Se estableció un valor inicial de $100 \mathrm{~kg}$ MS ha ${ }^{-1}$ para clases de sitio IV y V, $125 \mathrm{~kg} \mathrm{MS} \mathrm{ha}^{-1}$ en CS III y $150 \mathrm{~kg} \mathrm{MS} \mathrm{ha}^{-1}$ para clases de sitio I y II (Según datos de Peri 2009).

Tasa de crecimiento de biomasa (Tcrecbiom)

Aumento mensual de MS que surge de un crecimiento potencial afectado por variables ambientales a través de índices que lo limitan (ec. 5).

$$
\begin{aligned}
& \text { Tcrecbiom }=\text { Crecpot * IHS }-M S \text { *ITR }-M S \text { * } \\
& \text { ITA }-M S{ }^{*} I R m-M S
\end{aligned}
$$

Tasa de senescencia de biomasa (Tsenbiom)

Cantidad de MS que muere mensualmente y está afectada por la biomasa acumulada y por la humedad del suelo (ec. 6).

$$
\text { Tsenbiom }=\text { Isebiom } * \text { IHS }- \text { Se }
$$

\section{Crecimiento potencial (Crecpot)}

Máximo crecimiento que puede tener el pastizal en un mes si las condiciones ambientales no son limitantes. Se obtuvo a través de una función que incluye la biomasa acumulada (o inicial durante el primer mes) y un índice (entre 0 y 1 ) que contempla la CS del bosque (ec. 7).

$$
\begin{array}{r}
\text { Crecpot }=[((1 / \text { Biomacum }) * 2015,093)+1,999] \\
*(\text { ICS }-M S)
\end{array}
$$

Para el crecimiento potencial del pastizal se consideró un valor de 20 veces la biomasa 
inicial, tomado del trabajo de Gyenge et al. (2006) donde se midió el crecimiento relativo de Festuca pallescens en condiciones controladas. Considerando que el crecimiento potencial de un pastizal estará limitado por su crecimiento a través del tiempo debido a competencia por los recursos, cuya disponibilidad relativa disminuirá con su biomasa, se relacionaron datos de biomasa medidos a campo (Bahamonde et al. 2012a) en un mes determinado con su crecimiento al mes siguiente (en número de veces su biomasa). De esta manera asumiendo el crecimiento potencial en condiciones óptimas (Gyenge et al. 2006) y la variación del crecimiento relacionado a la biomasa inicial se ajustó una ecuación lineal inversa (primer término ecuación 7). El segundo término de la ecuación 7 representa la CS donde se desarrolla el bosque.

\section{Índice de CS para materia seca (ICS-MS)}

Toma valores entre 0 y 1 relacionando la clase de sitio del bosque de ñire (CS) con el crecimiento potencial del pastizal dentro del mismo. El factor para cada CS se obtiene de la siguiente función lineal:

$$
\text { ICS }-M S=(-0,19 * C S)+1,19
$$

Donde CS toma valores enteros de 1 a 5 (clasificación de Ivancich etal. 2011). La función fue generada a través de la comparación de datos de producción de MS en bosques de ñire creciendo en diferentes clases de sitio (Peri 2009; Bahamonde et al. 2012a).

Índice de humedad de suelo para materia seca (IHS-MS)

Relación entre la humedad volumétrica del suelo y el crecimiento potencial de las gramíneas, toma valores entre 0 y 1 (ec. 9).

$$
y=(0,019 x-0,007) / 1,386
$$

Donde $y$ es el IHS-MS, $x$ es la humedad volumétrica del suelo.

Índice de temperatura del aire para materia seca (ITA-MS)

Toma valores entre 0 y 1 , y relaciona la temperatura promedio mensual del aire con el crecimiento de las gramíneas (ec. 10).

$$
y=-0,002 x^{2}+0,09 x+0,037
$$

Siendo $x$ la temperatura media del aire mensual.

Esta función se tomó de Romera et al. (2009) quienes realizaron un ajuste a un modelo de crecimiento de praderas compuestas principalmente de gramíneas templadas (McCall \& Bishop-Hurley 2003), el cual estaba basado en mediciones realizadas en ambientes controlados.

Índice de transmisividad lumínica para materia seca (ITR-MS)

Indica el efecto de la apertura del dosel arbóreo sobre el crecimiento de la MS de gramíneas en el bosque de ñire. Toma valores de entre 0,24 y 1 , para aperturas de dosel de 0,1 y 1 (transmisividad $100 \%)$, respectivamente. Este índice surge de realizar comparaciones de producción máxima de MS de gramíneas en distintos niveles de transmisividad en bosques de ñire (y lugares aledaños sin árboles) en Patagonia Sur (Peri 2009; Bahamonde et al. 2012a).

Índice de efecto de radiación mensual sobre materia seca (IRm-MS)

Representa la variación mensual de la radiación fotosintéticamente activa y su efecto sobre la producción de MS. Toma valores entre 0 y 1 , siendo 1 el mes de máxima radiación y los restantes meses toman valores proporcionales al máximo. El índice se basa en los valores de radiación fotosintéticamente activa estimados a través de fotografías hemisféricas (Bahamonde 2011) (Figura S3).

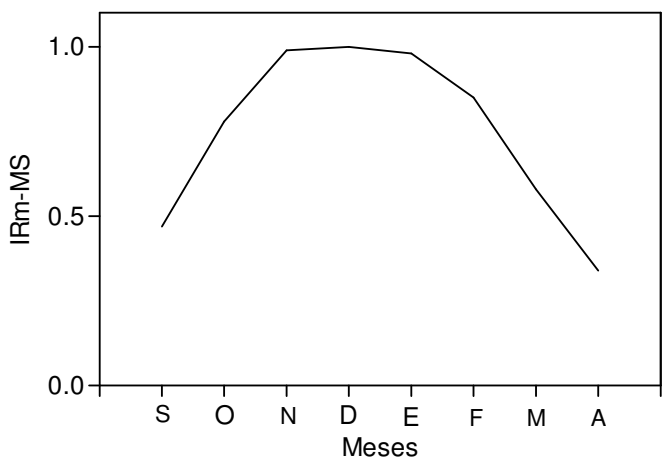

Figura S3. Índice del efecto de la radiación fotosintéticamente activa total mensual (directa más difusa) sobre la producción de materia seca (IRm-MS) para un modelo de simulación de gramíneas creciendo en sistemas silvopastoriles en bosques de N. antarctica.

Figure S3. Index of the effect of the photosynthetically active radiation on dry matter production (IRm-MS) for a simulation model of grasses growing in $N$. antarctica forests under silvopastoral use.

\section{Índice de senectud por biomasa (ISebiom)}

Indica la senescencia de las gramíneas creciendo en SSP basado en una proporción de la MS acumulada y un índice del efecto de la humedad volumétrica del suelo sobre la senescencia (ec. 11). 
ISebiom $=($ Biomacum $*(0,2$ ó 0,36) $) *$ IHS - Se [11]

Los valores varían entre 20 y $36 \%$ de senescencia mensual y surgieron de datos de senescencia de gramíneas creciendo en SSP con ñire cuando otros factores ambientales no eran limitantes (Bahamonde et al. 2012a).

Índice de efecto de humedad de suelo sobre senescencia (IHS-Se)

Se refiere al aumento lineal de la senescencia de las gramíneas cuando la humedad del suelo cae por debajo de un valor umbral como proporción de la humedad a capacidad de campo. Se estableció empíricamente un valor de 20\% de humedad como umbral (Bahamonde 2011). En este caso el modelo trabaja con un valor mínimo de $10 \%$ de humedad volumétrica, y por lo tanto se usa un aumento lineal de la senescencia de hasta 1,5 veces su valor original $(0,2$ ó 0,36$)$ cuando la humedad volumétrica disminuye de 20 a $10 \%$.

\section{PB en biomasa}

PB (\%) mensual durante la temporada de crecimiento de gramíneas creciendo en SSP y está regulada por un incremento y una disminución mensual $(\mathrm{dt})$ relacionados a las condiciones ambientales en las que crecen (ec. 12). El modelo usa un valor inicial de $8 \%$, el cual asume que al inicio de la temporada de crecimiento el pastizal tiene una concentración inicial de $\mathrm{N}$ adecuada para su crecimiento (Taiz \& Zeiger 2006) y coincide con los valores encontrados en gramíneas en Patagonia Sur (Peri \& Lasagno 2010).

PBenbiomasa $=$ PBenbiomasa $(t-d t)+($ Inc PB en biom - Dism PB en biom $) * d t$

Incremento de PB en biomasa (Inc PB en biom)

Aumento de PB a través del tiempo dado por su valor potencial y los efectos de las variables ambientales (ec. 13).

Inc PB en biom $=P B$ Potencial * ITA-PB * ITS$P B{ }^{*} I H S-P B{ }^{*} I A D-P B$

Donde ITA es índice de temperatura de aire; ITS es índice de temperatura de suelo; IHS es índice de humedad del suelo; IAD es índice de apertura del dosel arbóreo.

\section{PB Potencial}

Valor de PB máximo mensual encontrado para gramíneas creciendo en SSP de ñire (Bahamonde et al. 2012a) en la zona de influencia del modelo, el cual fue de
$22 \%$. Esto no implica que este sea el valor máximo alcanzable, ya que dependerá de las condiciones ambientales que el modelo considera influyentes para su variación.

Índice de temperatura del aire sobre PB (ITA-PB)

Efecto de la temperatura del aire sobre la $\mathrm{PB}$, toma valores entre 0,5 y 1 . Este índice se generó estandarizando a 1 el valor máximo de PB obtenido de una regresión no lineal entre datos de temperatura de aire y $\%$ PB medidos en 5 sitios en Patagonia Sur (Bahamonde et al. 2012a).

Índice de temperatura del suelo sobre PB (ITS-PB)

Efecto de la temperatura del suelo sobre la concentración de PB basado en la relación empírica obtenida con datos del trabajo de Bahamonde et al. (2012a) (ec. 14).

$$
y=\left(0,069 x^{2}-1,77 x+19,55\right) / 17,85
$$

Donde $y$ es el índice, $x$ es la temperatura del suelo.

Índice de humedad del suelo sobre PB (IHS-PB)

Efecto de la humedad volumétrica del suelo (en los primeros $30 \mathrm{~cm}$ ) sobre la PB de las gramíneas tomando valores de entre 0,48 y 1 para el rango de humedad volumétrica del suelo que usa el modelo (Bahamonde 2011).

Índice de apertura de dosel sobre \% de PB (IAD-PB)

Señala el efecto de la apertura del dosel arbóreo sobre la PB, toma valores entre 0,5 y

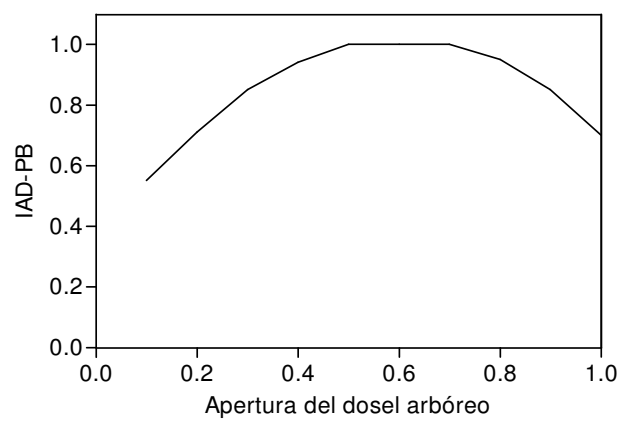

Figura S4. Relación entre la apertura del dosel arbóreo y el índice de apertura del dosel sobre la concentración de proteína bruta (IAD-PB) para un modelo de simulación de gramíneas creciendo en sistemas silvopastoriles en bosques de $N$. antarctica. Ecuación 15.

Figure S4. Relationship between the canopy openness and the index of the canopy openness on crude protein concentration (IAD-PB) for a simulation model of grasses growing in N. antarctica forests under silvopastoral use. 
1 , siendo más alto a valores intermedios de apertura de dosel (Figura S4, ecuación 15). Este índice se basó en la relación obtenida de datos de concentración de PB de gramíneas creciendo en distintos niveles de apertura de dosel arbóreo de bosques de ñire (Bahamonde 2011) donde se seleccionaron y compararon valores de $\mathrm{PB}$ en meses en que otras variables ambientales como temperaturas de aire y suelo, y humedad volumétrica del suelo fueron similares entre distintas coberturas de copa para reducir el efecto que dichas variables podrían tener en el \% de PB.

$$
\mathbf{y}=\left(-29,54 x^{2}+34,56 x+4,48\right) / 14,6
$$

Disminución de PB en biomasa (Dism PB en biom)

Variación de la PB en el tiempo, lo cual genera una disminución que depende del tiempo transcurrido y representa una proporción de la PB acumulada (ec. 16).

Dism PB en biom $=(1-(E f T-P B)) * P B$ en biom [16]

Efecto del tiempo sobre PB (EfT-PB)

Relaciona el número de días con la variación de $\mathrm{PB}$, tomando un valor de 1 al comienzo del periodo de crecimiento, disminuyendo posteriormente hasta los 150 días cuando nuevamente comienza a aumentar. Se generó estandarizando al valor máximo encontrado con datos medidos a campo ( $22 \%$ ) y el ajuste de una función polinomial (Bahamonde 2011) (ec. 17).

$y=\left(1,8 * 10^{-6} x^{3}+0,002 x^{2}-0,468 x+32,91\right) / 22[17]$

Donde $y$ es el índice del efecto del tiempo sobre la $\mathrm{PB}, x$ es el número de días. 